\title{
In-situ Measurement via E/M Impedance Spectroscopy Technique Using Shear Horizontal Piezoelectric Wafer Active Sensors
}

\author{
Tuncay Kamaş** \\ *Department of Mechanical Engineering, Faculty of Engineering and Architecture, Eskişehir Osmangazi University, Meselik \\ Campus Odunpazarı Eskişehir \\ tkamas@ogu.edu.tr \\ ${ }^{\ddagger}$ Corresponding Author; Tuncay Kamaş, Eskişehir Osmangazi University Meselik Campus Engineering and Architecture \\ Faculty M2 Block Mechanical Engineering Dept. C305 Odunpazarı, Eskişehir, Tel: +90 2222393750 (3367), \\ tkamas@ogu.edu.tr
}

Received:26.05.2015 Accepted:15.06.2015

\begin{abstract}
In this paper, an electromechanical in-situ measurement method is to be developed for determination of some properties of fuel-oils. Electromechanical impedance spectroscopy (EMIS) method is to be utilized in the measurement technique which will be developed. This method uses piezoelectric sensors that work in electromechanical principles as resonator and dynamically determines the characteristics of a medium that the resonator interacts. EMIS method has been mostly used to determine the dynamic changes in elastic properties of solid materials. This method utilizes the shear horizontal piezoelectric wafer active sensor (SH-PWAS) to generate shear horizontal mode standing waves. Piezoelectric wafer active sensors (PWAS), that are to be utilized in this study, have recently been developed as easily manufactured, small in size and weight, and broad banded transducers to utilize in qualitative and quantitative measurement systems.
\end{abstract}

Keywords E/M impedance, shear horizontal, piezoelectricity, fuel

\section{Introduction}

Piezoelectric wafer active sensor (PWAS) is lightweighted, inexpensive, unobtrusive, minimally intrusive sensor requiring low-power [1]. PWAS is made of piezoelectric ceramic with electric field polarization across the electrodes deposited on both surfaces. It has recently been extensively employed in many applications for in-situ structural inspection in fields such as nuclear plants, structural health monitoring (SHM) and non-destructive evaluations (NDE). The SHM methods include techniques through guided wave generation/ transduction and electromechanical impedance spectroscopy (EMIS). EMIS techniques where PWAS is employed as a resonator are used to generate standing waves in local field of a substrate structure. EMIS method has been utilized to determine the local dynamic characteristics of PWAS bonded on a host structure for in-situ ultrasonic. [2]-[4], and [5] utilized the EMIS method for high frequency local modal sensing. Beside these authors, many rigorous modelling attempts exist in the literature regarding different aspects of impedance and admittance spectra of bonded piezoelectric transducers [6][10] in the last decade.
Conventional PWAS is thin rectangular or circular wafer that is poled in thickness direction, with electrodes on top and bottom surfaces; those types of PWAS are either used in in-plane or thickness mode [11]. In in-plane mode, applying an electric field in thickness direction $E_{3}$ causes the sensor lateral dimensions to increase or decrease, a longitudinal strain will occur $\varepsilon_{1}=d_{13} E_{3}$ where $d_{13}$ is the piezoelectric coupling coefficient measured in $[\mathrm{m} / \mathrm{V}]$. Thickness mode is a mode that occurs simultaneously with extension mode, but dominates at higher frequencies in $\mathrm{MHz}$, in which strain in the thickness direction will occur $\varepsilon_{3}=d_{33} E_{3}$, where $d_{33}$ is the piezoelectric coupling coefficient in thickness direction [12]. A different mode of oscillation can be achieved when the applied electric field is applied perpendicular to the poling direction; and it is referred as shear mode. For most piezoelectric materials, the coupling coefficients associated with shear mode have the largest value of all coefficients [13]. The higher values of shear coupling coefficients make SH-PWAS superior in actuation and sensing. SH waves are also preferable because the first symmetric mode is nondispersive, i.e. wave speed is constant at different frequencies. On the other hand, one of the important disadvantages of SH-PWAS is that thicker transducers are 
needed to sustain and generate the shear actuation and due to high density of piezo-ceramic materials $\left(\approx 7600 \mathrm{~kg} / \mathrm{m}^{3}\right.$ for APC850 piezo-ceramic Navy II type); using of shear mode piezoelectric elements increases the mass of the system considerably.

Kanazawa and Gordon [14] proposed an analytical definition of the resonance frequency shift by purely mechanical analysis which coupled the standing shear wave in the quartz to a damped propagating shear wave in Newtonian fluid i.e.

$$
\Delta f=-f_{o}^{3 / 2} \sqrt{\eta_{L} \rho_{L} / \pi \mu_{q} \rho_{q}}
$$

and verified by the experimental results in terms of the changes in resonance frequency of the quartz resonator whose one surface is in contact with water that owned varying concentration of glucose and ethanol. In the paper, the boundary layer was identified as the characteristic length of exponentially decaying viscous effects of the liquid on the resonance frequency because the displacement exponentially dies out in the liquid. The approach has been applied for quartz crystal resonators with overlying viscous liquids, thin elastic films and viscoelastic layers [15]-[18]. However the theoretical method derived by Kanazawa \& Gordon is only valid for overlying viscous fluid of infinite extent therefore the method analyzes bulk acoustic waves (BAW) which remains the sensor sensitivity low. The sensors utilizing surface acoustic waves (SAW) are superior to the conventional BAW devices in liquid sensor applications since SAW devices can operate at much higher frequencies and more mass sensitive since SAW possesses large attenuation and energy loss due to a mode conversion in the liquid and dissipates due to the viscous effects [15]. Guo \& Sun [19] found that shear type SAW is a good candidate for liquid sensing applications. Other alternative to the SAW presented was the shear horizontal $(\mathrm{SH})$ surface wave as seen in Fig. 1.

Kamal et-al. [13] developed a one dimensional analytical model for EMIS of SH-PWAS (Fig. 2) under stress-free boundary condition using the resonator theory with constant electric field assumption as seen in Eq. (2) that defines the $\mathrm{E} / \mathrm{M}$ admittance of free SH-PWAS. They verified the analytical model by finite element simulation and experimental EMIS measurement as seen in Fig. 3.

$$
Y=\frac{\hat{I}}{\hat{V}}=i \omega C\left[1-\kappa_{35}^{2} \frac{1}{\phi \cot \phi}\right]^{-1}
$$

where $\kappa_{35}$ is the shear horizontal electromechanical coupling coefficient and can be defined as

$$
\kappa_{35}=\sqrt{\frac{d_{35}^{2}}{\varepsilon_{33}^{T} s_{55}^{E}}}
$$

$\phi$ is the product of the half of the wave number and the characteristic length of PWAS i.e. $\phi=\gamma l / 2$ and $C$ is the capacitance of PWAS.

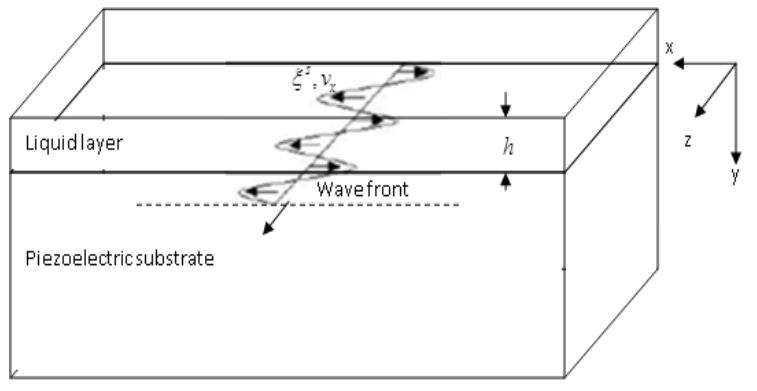

Fig. 1 Schematic of the propagation of a Shear horizontal (SH) wave along the interface between a piezoelectric substrate and a liquid layer

In this paper, we present one-dimensional analytical model for EMIS of SH-PWAS in contact with different liquids in different density and viscosity. The EMIS of SHPWAS with different fuels are also simulated by using 3-D coupled field finite element models in ANSYS. The material properties of fuels were published in the paper by Schaschke et-al. [20]. The published fuel material properties at room temperature $\left(25^{\circ} \mathrm{C}\right)$ and at the atmospheric pressure $(1 \mathrm{~atm})$ are used in the simulations in order to obtain some preliminary results before the experimental studies. The trends of the E/M impedance and admittance simulation results are discussed by comparing the fuels with different viscosity and density. The experimental results will not be presented in this paper.

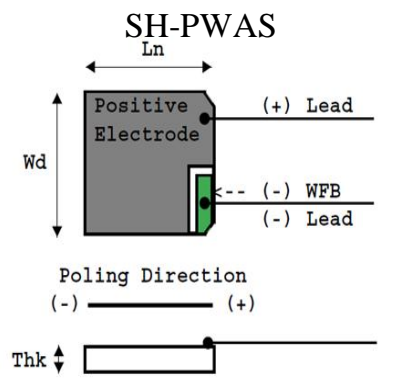

Fig. 2 Schema of shear horizontal piezoelectric wafer active sensor under stress-free boundary condition

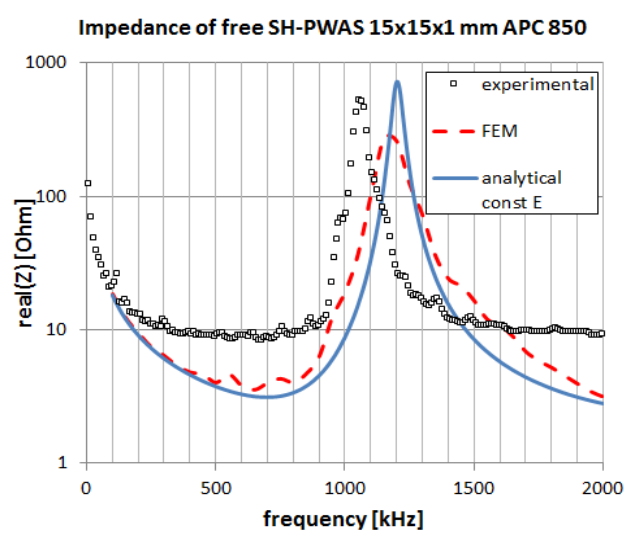

Fig. 3 Real part of impedance for APC850 type free SHPWAS in sizes of $15 \mathrm{mmx} 15 \mathrm{mmx} 1 \mathrm{~mm}$ [13] 


\section{Analytical modelling of SH-PWAS in contact with a fuel layer}

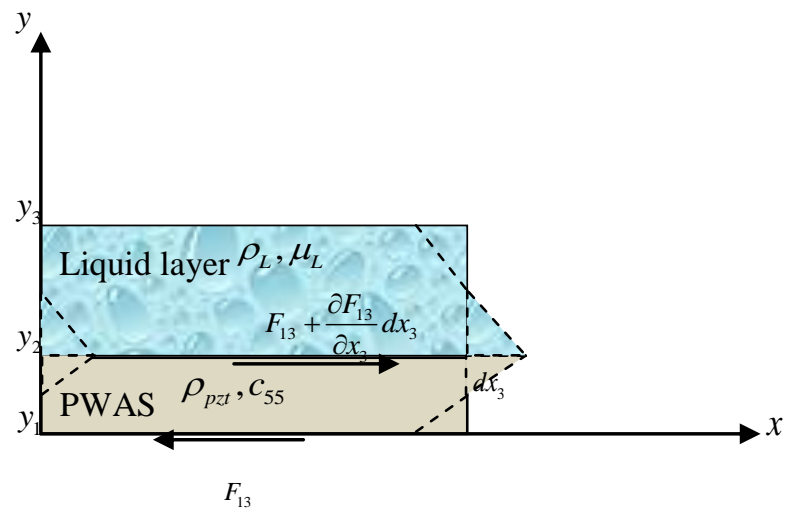

Fig. 4 Schematic of piezo-wafer resonator deformed in shear horizontal $d_{35}$ mode by induced $T_{5}=T_{13}$ shear stress

Table 1 Properties of APC 850 piezoelectric ceramic [22]

\begin{tabular}{|c|c|c|c|c|c|c|}
\hline Property & $\begin{array}{c}\rho \\
k g / m^{3}\end{array}$ & $\begin{array}{l}d_{33} \\
m / V\end{array}$ & $\begin{array}{l}d_{31} \\
m / V\end{array}$ & $\begin{array}{c}g_{33} \\
V m / N\end{array}$ & $\begin{array}{c}g_{31} \\
V m / N\end{array}$ & $\begin{array}{c}s_{33}^{E} \\
m^{2} / N\end{array}$ \\
\hline APC 850 & 7700 & $400 \times 10^{-12}$ & $\underset{12}{175 \times 10^{-}}$ & $26 \times 10^{-3}$ & $12.4 \times 10^{-3}$ & $\begin{array}{r}17.3 \\
\times 10^{-12}\end{array}$ \\
\hline Property & $\begin{array}{c}S_{11}^{E} \\
m^{2} / N\end{array}$ & $\varepsilon_{33}^{T} / \varepsilon_{0}$ & $\kappa_{p}$ & $\kappa_{33}$ & $\kappa_{31}$ & $v$ \\
\hline APC 850 & $\begin{array}{c}15.3 \\
\times 10^{-12}\end{array}$ & 1750 & 0.63 & 0.72 & 0.36 & 0.35 \\
\hline
\end{tabular}

In this section, the response of a piezo-resonator coupled with liquid layer (Fig. 4) is presented in terms of E/M impedance and admittance spectra. The shear horizontal waves and the corresponding piezoelectric constitutive equations were taken into account to derive the EMIS response of a SH-PWAS resonator in contact with liquid layer [21].

The 1-D analytical SH-PWAS EMIS model including the liquid layer effects was derived. The particle velocity of SH-PWAS along its thickness in contact with water can be seen in Fig. 5(a). The particle velocity at the interface is $1.332 \mu \mathrm{m} / \mathrm{sec}$ at electric field amplitude of $20 \mathrm{kV} / \mathrm{m}$ and $\mathrm{SH}-$ PWAS sizes are $15 \mathrm{~mm} \times 15 \mathrm{mmx} 1 \mathrm{~mm}$. The particle velocity of SH-PWAS along its thickness in contact with water and ethylene glycol can be seen in Fig. 5(b), the particle velocity at the interface between PWAS and ethylene glycol is 0.4702 $\mu \mathrm{m} / \mathrm{sec}$. This shows that the velocity continuity at the interface was satisfied. Piezoelectric material properties given in Table $\mathbf{2}$ are used for the calculations.

The liquid particle velocity can be defined by

$$
v_{L}=i \omega\left\{\theta \psi-e^{-\phi_{S H}}\right\} \frac{u_{I S A}}{\phi_{S H}} e^{i \gamma_{L}\left(x_{3}-y_{2}\right)}
$$

PWAS particle displacement is
$u_{1}\left(x_{3}\right)=\left\{\theta\left(e^{-\phi_{S H}\left(\frac{x_{3}-y_{1}}{h_{p}}+1\right)}+e^{\phi_{S H}\left(\frac{x_{3}-y_{2}}{h_{P}}\right)}\right)-e^{-\phi_{S H}\left(\frac{x_{3}-y_{1}}{h_{p}}\right)}\right\} \frac{u_{I S A}}{\phi_{S H}}$

Where,

$$
\begin{aligned}
& \theta=\frac{\left[\lambda \omega h_{p} e^{-\phi_{S H}}-\phi_{S H} \chi\right]}{\left[\phi_{S H} \xi+\lambda \omega h_{p} \psi\right]} \\
& \lambda=s_{55}^{E} \eta_{L} \gamma_{L} \\
& \xi=-e^{-2 \phi_{S H}}+1 \\
& \psi=e^{-2 \phi_{S H}}+1 \\
& \chi=e^{-\phi_{S H}}-1 \\
& \phi_{S H}=i \gamma h_{p}
\end{aligned}
$$

and the induced displacement is introduced as

$$
u_{I S A}=d_{35} \hat{E}_{3} h_{p}
$$

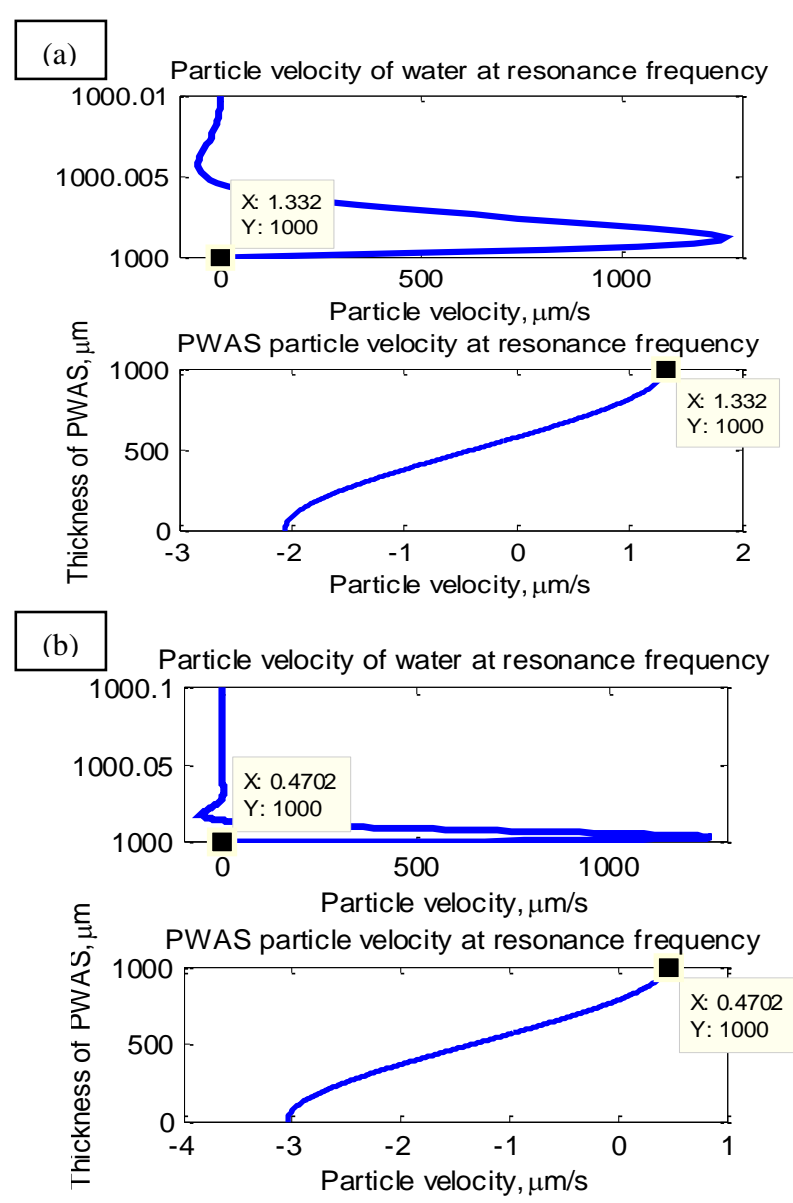

Fig. 5 Liquid particle velocity change in the vicinity of PWAS-liquid interface at the first resonance frequency of $850.4 \mathrm{kHz}$ (a) for water with the density of $1000 \mathrm{~kg} / \mathrm{m}^{3}$ and the dynamic viscosity of $8.9 \times 10^{-4}$ Pa.s (b) for ethylene glycol with the density of $1113 \mathrm{~kg} / \mathrm{m}^{3}$ and the dynamic viscosity of

0.01 Pa.s. PWAS sizes $15 \mathrm{~mm} \times 15 \mathrm{mmx} 1 \mathrm{~mm}$ 
Table 2 Material properties of fuels used in analytical simulations

\begin{tabular}{l|lc}
\hline Fuel Type & Density $\mathrm{kg} / \mathrm{m}^{3}$ & $\begin{array}{l}\text { Viscosity } \\
x 10^{-3} \text { Pa.s }\end{array}$ \\
\hline Fuel 1 & 725 & 2 \\
Fuel 2 & 725 & 5 \\
Fuel 3 & 925 & 2 \\
\hline
\end{tabular}

$$
Y=i \omega C_{0}\left\{1-\kappa_{35}^{2}+\frac{\kappa_{35}^{2}}{\phi_{S H}}\left[\theta\left(e^{-\phi_{S H H}\left(\frac{x_{3}-y_{1}}{h_{p}}+1\right)}+e^{\phi_{S H}\left(\frac{x_{3}-y_{2}}{h_{p}}\right)}\right)-e^{-\phi_{S H}\left(\frac{x_{x^{\prime}}-y_{1}}{h_{p}}\right)}\right]\left(\xi-2 e^{-\phi_{S H H}}\right)\right\}
$$

In the analytical simulation, first EMIS of SH-PWAS under stress-free boundary condition was simulated. Then two liquids with different density and viscosity are used as a layer on top of SH-PWAS such as water and fuel oil as seen in the model (Fig. 4) and derived as shown in Eq.(8).
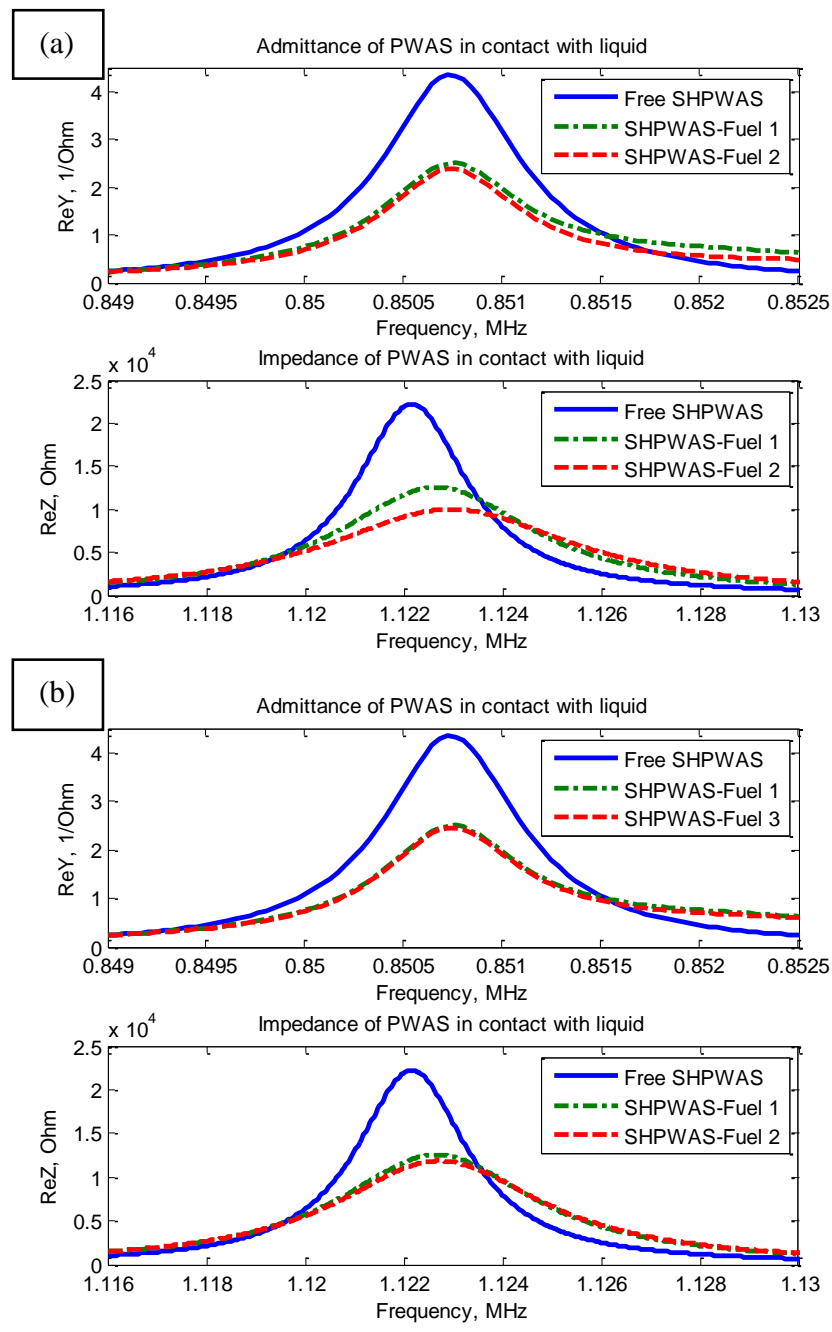

Fig. 6 Analytical simulation results for real and imaginary parts of E/M impedance and admittance of SHPWAS interacting with fuels (a) in different viscosity and (b) in different density
The density and the viscosity of fuels are listed in Table 2. The analytical simulation results at the first shear horizontal mode of SH-PWAS are presented in Fig. 6(a) in terms of real part of E/M impedance and admittance of SHPWAS and SH-PWAS coupled with fuel 1 and fuel 2 . The fuels 1 and 2 have the same density but different viscosity. The results presented in Fig. 6(b) are also EMIS of SHPWAS and SH-PWAS coupled with fuel 1 and fuel 3. The fuels have the same viscosity but different density. In the E/M impedance results, one can observe that the amplitude shifts down whereas the anti-resonance frequency shifts up as the fuel viscosity increases; however as the density of the fuel layer is increased from $725 \mathrm{~kg} / \mathrm{m}^{3}$ to $925 \mathrm{~kg} / \mathrm{m}^{3}$, the amplitude of impedance peak slightly decreases. Admittance results do not hold promise for good indication of parameters changes because only the amplitude of the real part of the admittance decreases when the liquid layer existed and as the viscosity was increased but the frequency did not noticeably change. Also, as the density of fuel was increased, we observed very little change in the amplitude of the admittance.

\section{Coupled Field FEA Modelling of SH-PWAS in contact with a fuel layer}

\subsection{Free SH-PWAS}
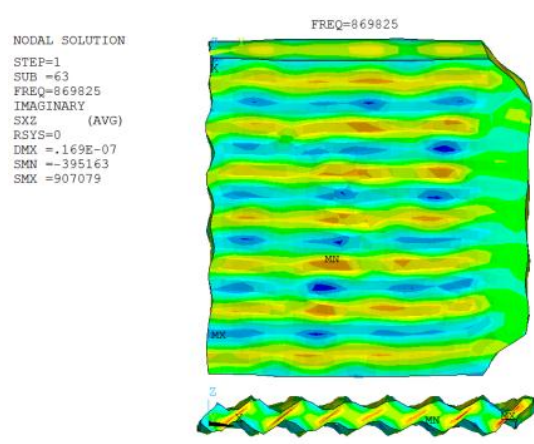

(a)

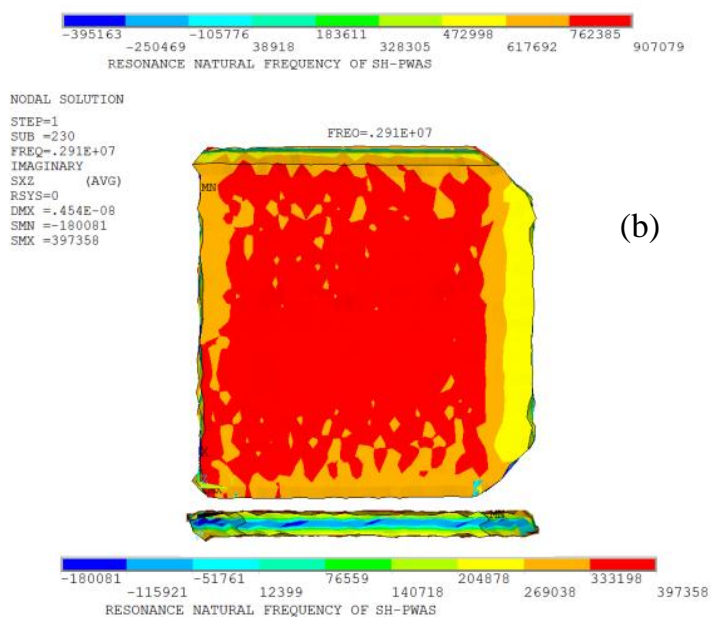

Fig. 7 (a) The first shear horizontal mode shape of SHPWAS at the resonance frequency of $869 \mathrm{kHz}$. (b) The second shear horizontal mode shape of SH-PWAS at the resonance frequency of $2.91 \mathrm{MHz}$. The counter plots show $\mathrm{T}_{\mathrm{xz}}$ shear stress distribution 


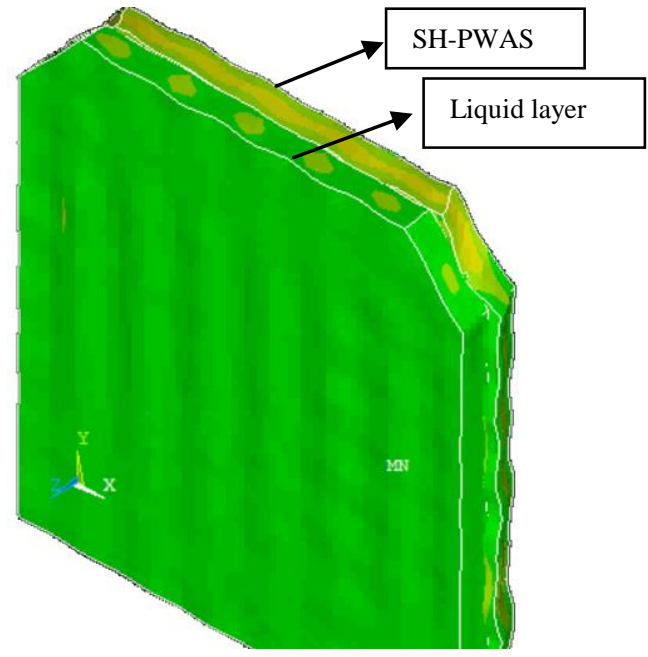

Fig. 8 Coupled field FEA model of SH-PWAS with liquid layer vibrating in the first shear horizontal mode

We first modelled three dimensional (3-D) $15 \mathrm{mmx} 15 \mathrm{mmx} 1 \mathrm{~mm}$ SH-PWAS under traction-free boundary conditions in ANSYS $^{\circledR}$ without introducing the liquid layer; we used APC 850 ceramic piezoelectric material properties that were presented in Table 2. ANSYS multi-physics software with the implicit solver was used to perform EMIS computation in frequency domain. To perform the coupled stress and electric field analysis of PWAS transducers, coupled field piezoelectric elements were used. These coupled field finite elements consist of both mechanical and electrical fields. The elements that represent piezoelectric effects in our analysis are the 3-D coupled field solid elements i.e. SOLID5. The electrical charge $Q$ is the electrical reaction corresponding to the voltage DOF. The charge $Q$ is then used to calculate the admittance and impedance data. The admittance $Y$ is calculated as $I / V$, where $I$ is the current in ampere and $V$ is the applied potential voltage in volts. The current comes from the charge accumulated on the PWAS surface electrodes and is calculated as

$$
I=i \omega \sum Q_{i}
$$

with $\omega$ being the operating frequency, $i$ is the complex number, and $\sum Q_{i}$ is the summed nodal charge.

$$
\left[C_{p}\right]=\left[\begin{array}{cccccc}
97 & 49 & 49 & 0 & 0 & 0 \\
49 & 97 & 44 & 0 & 0 & 0 \\
49 & 49 & 84 & 0 & 0 & 0 \\
0 & 0 & 0 & 24 & 0 & 0 \\
0 & 0 & 0 & 0 & 22 & 0 \\
0 & 0 & 0 & 0 & 0 & 22
\end{array}\right] \mathrm{GPa}
$$

$$
\begin{aligned}
& {\left[\varepsilon_{p}\right]=\left[\begin{array}{ccc}
947 & 0 & 0 \\
0 & 605 & 0 \\
0 & 0 & 947
\end{array}\right] \times 10^{-8} \mathrm{~F} / \mathrm{m}} \\
& {\left[e_{p}\right]=\left[\begin{array}{cccccc}
0 & 0 & 0 & 0 & 12.84 & 0 \\
0 & 0 & 0 & 12.84 & 0 & 0 \\
-8.02 & -8.02 & 18.31 & 0 & 0 & 0
\end{array}\right] \mathrm{C} / \mathrm{m}^{2}}
\end{aligned}
$$

where $\left[C_{p}\right]$ is the stiffness matrix, $\left[\varepsilon_{p}\right]$ is the dielectric matrix, and $\left[e_{p}\right]$ is the piezoelectric matrix. The density of the PWAS material is assumed to be $\rho=7700 \mathrm{~kg} / \mathrm{m}^{3}$.

The first and second shear horizontal mode shapes are shown in Fig. 7(a) and (b) respectively. We also retained the electromechanical impedance and admittance results from the free SH-PWAS simulation.

\subsection{SH-PWAS in contact with compressible and inviscid fluid}

We introduced the liquid layer in the same geometric shape and sizes by using the FLUID30 element type for 3-D liquid layer modelling merged with the SH-PWAS elements (Fig. 8). One restriction that this fluid element has is that the dissipative effect due to the fluid viscosity was neglected. However, the sound absorption at the interface was taken into account by generating a damping matrix using the surface area and boundary admittance at the interface. The boundary admittance (MU) can be input as material property between 0 and 1 . The reference pressure (PREF) was used to calculate the sound pressure level of element (defaults to $20 \times 10^{-6} \mathrm{~N} / \mathrm{m}^{2}$ ). The speed of sound

$$
c=\sqrt{k / \rho_{0}}
$$

in the fluid can be input by SONC where $k$ is the bulk modulus of the fluid and $\rho_{0}$ is the mean fluid density (input as DENS) [23].

In order to show the effect of the existence of a liquid layer on EMIS of SH-PWAS, we illustrate the preliminary results in terms of a wide band impedance spectra by $\mathrm{CF}$ FEA analysis including in-plane modes between $100 \mathrm{kHz}$ and $800 \mathrm{kHz}$; thickness modes in relatively high frequency range of $\mathrm{MHz}$; as well as three shear horizontal modes that appear at anti-resonance frequency of around $1 \mathrm{MHz}, 3 \mathrm{MHz}$, and $4.5 \mathrm{MHz}$ (Fig. 9). It can be clearly observed that amplitude and frequency shift due to the presence of the water layer was accomplished in the FEA simulations. As seen in the analytical simulations in the preceding section, only amplitude shift occurs in admittance spectra due to the water layer existence but not frequency shift. In FEA, we adopted the fuel material properties from Reference [24] as provided in Table 2. 

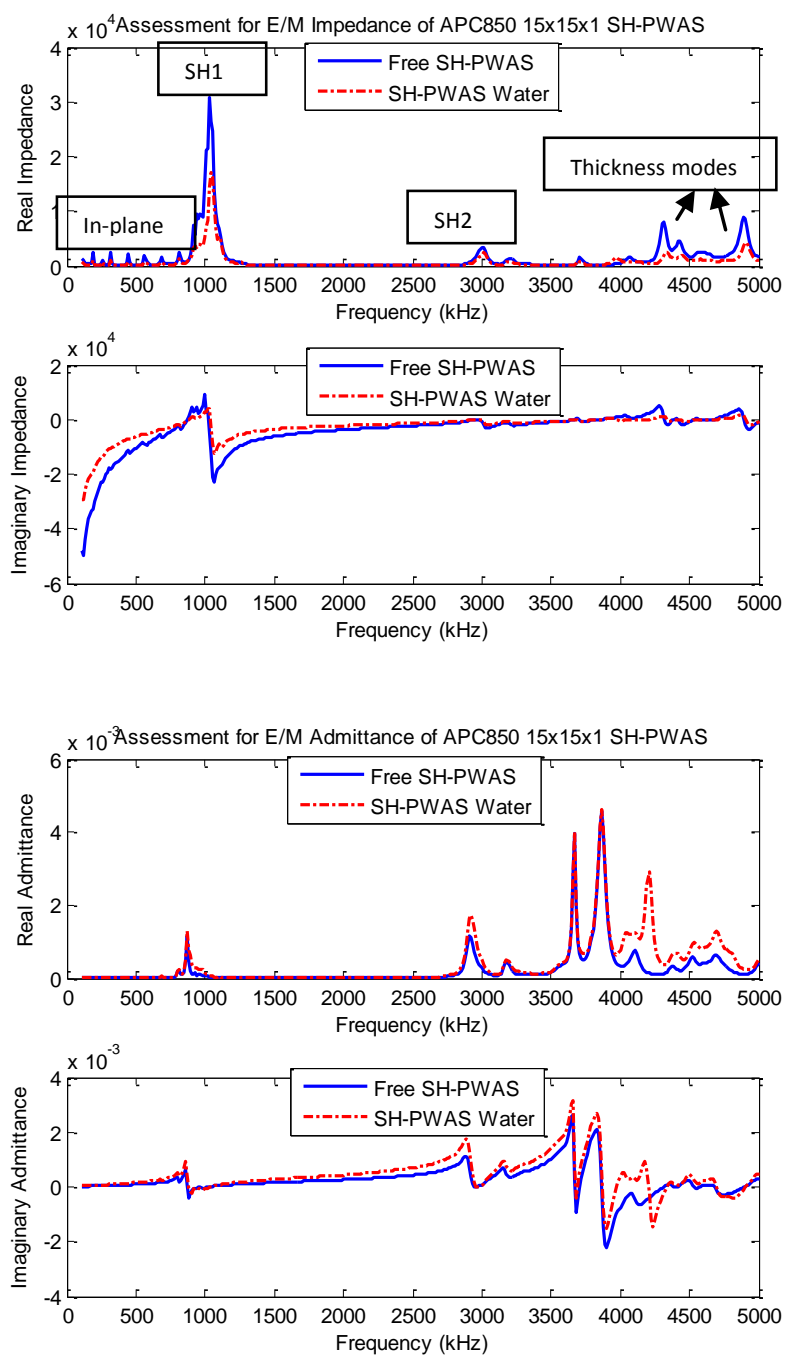

Fig. 9 Electromechanical impedance (left) and admittance (right) spectra of $15 \mathrm{~mm} \times 15 \mathrm{~mm} \times 1 \mathrm{~mm}$ SH-PWAS and SH-PWAS coupled with a $1 \mathrm{~mm}$ thick layer of water

(Please use two-column publishing format)
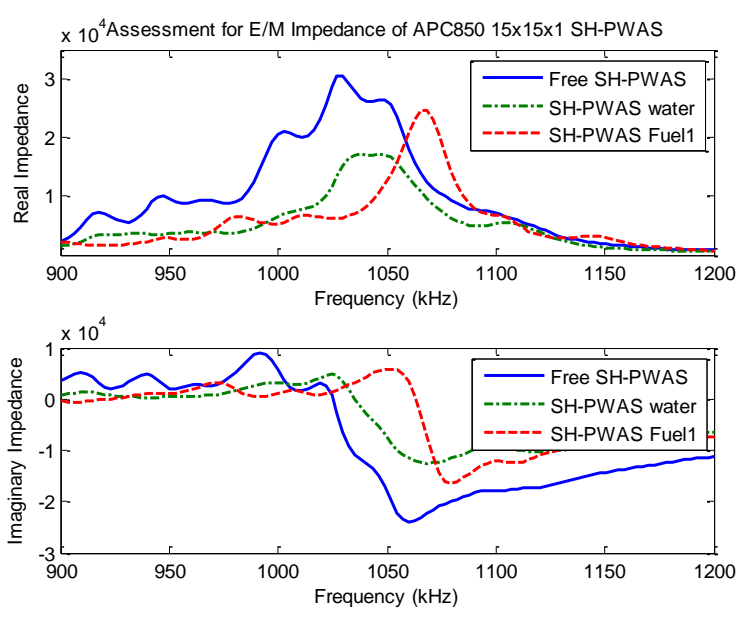

Fig. 10 Electromechanical impedance spectra of $15 \mathrm{mmx} 15 \mathrm{mmx} 1 \mathrm{~mm}$ SH-PWAS; SH-PWAS coupled with a $1 \mathrm{~mm}$ thick layer of water; and SH-PWAS coupled with a $1 \mathrm{~mm}$ thick layer of fuel-oil
The CF-FEA simulations also proves that E/M impedance spectrum is better indicator of presence of liquid layer and the changes in the liquid layer in comparison to the admittance spectrum. From this point of view, we focus on E/M impedance simulation results in our FEA analyses. The next results were found to be a good indicator of the liquid density effects on EMI spectrum. Since the fuel 1 is less dense $\left(825 \mathrm{~kg} / \mathrm{m}^{3}\right)$ than the water $\left(1000 \mathrm{~kg} / \mathrm{m}^{3}\right)$, the amplitude of impedance peak of SH-PWAS coupled with the fuel 1 is higher than that of the SH-PWAS with water.

\section{Summary and Conclusion}

In this paper, we presented the E/M impedance spectroscopy of piezoelectric wafer active resonator capable of being excited in shear horizontal modes (SH-PWAS) with relatively high electromechanical coupling coefficient. We developed and simulated one-dimensional analytical model and three dimensional coupled field finite element models for SH-PWAS coupled with liquid layer as well as SH-PWAS under stress-free boundary condition. We analysed the results from both analytical and CF-FEA simulations for both free SH-PWAS and SH-PWAS interacting with different liquids and fuel-oils. We aimed at developing new methodology for measuring the fuel-oil material properties such as viscosity and density by measuring the E/M impedance and admittance of SH-PWAS in contact with different liquids with different viscosity and density. The results from both simulations showed that E/M impedance is a good indicator to develop the new measurement technique. This paper presents preliminary study results and needs further developments and investigations to develop the complete methodology of fueloil parameter measurement.

\section{Acknowledgements}

Acknowledgments are thankfully sent to Prof. Victor Giurgiutiu and Dr. Abdelrahman Kamal for their support to this research. 


\section{References}

[1] V. Giurgiutiu, J. Bao, and W. Zhao, "Active Sensor Wave Propagation Health Monitoring of Beam and Plate Structures," in Proc of SPIE's 8th International Symposium on Smart Structures and Materials, 2001, no. March.

[2] C. Liang, F. P. Sun, and C. a. Rogers, "Coupled Electro-Mechanical Analysis of Adaptive Material Systems -- Determination of the Actuator Power Consumption and System Energy Transfer," J. Intell. Mater. Syst. Struct., vol. 5, no. 1, pp. 12-20, Jan. 1994.

[3] F. P. Sun, C. Liang, and C. A. Rogers, "Structural modal analysis using collocated piezoelectric actuator/sensors: an electromechanical approach," in Proc. SPIE 2190, Smart Structures and Materials 1994: Smart Structures and Intelligent Systems, 238, 1994.

[4] A. N. Zagrai and V. Giurgiutiu, "Electro-Mechanical Impedance Method for Damage Identification in Circular Plates," vol. 40, 2001.

[5] G. Park, H. Sohn, C. R. Farrar, and D. J. Inman, "Overview of Piezoelectric Impedance-Based Health Monitoring and Path Forward," Shock Vib. Dig., vol. 35, no. 6, pp. 451-463, Nov. 2003.

[6] S. Bhalla, P. Kumar, A. Gupta, and T. K. Datta, "Simplified Impedance Model for Adhesively Bonded Piezo-Impedance Transducers," J. Aerosp. Eng., vol. 22, no. 4, pp. 373-382, Oct. 2009.

[7] H. A. Tinoco and A. L. Serpa, "Bonding influence on the electromechanical admittance of piezoelectric sensors bonded to structures based on EMI technique," 2011.

[8] V. G. Annamdas and M. a. Radhika, "Electromechanical impedance of piezoelectric transducers for monitoring metallic and non-metallic structures: A review of wired, wireless and energyharvesting methods," J. Intell. Mater. Syst. Struct., vol. 24, no. 9, pp. 1021-1042, Mar. 2013.

[9] S. Srivastava, S. Bhalla, A. Madan, and A. Gupta, "Biomedical Applications of Smart Piezoelectric Materials," in 9th International Symposium on Advanced Science and Technology in Experimental Mechanics, 2014, pp. 9-12.

[10] Y. Y. Lim and C. K. Soh, "Towards more accurate numerical modeling of impedance based high frequency harmonic vibration," Smart Mater. Struct., vol. 23, no. 3, p. 035017, Mar. 2014.

[11] T. Kamas, B. Lin, and V. Giurgiutiu, "Analytical modeling of PWAS in-plane and out-of-plane electromechanical impedance spectroscopy (EMIS)," in SPIE Smart Structure and Materials + Nondestructive Evaluation and Health Monitoring 2013, Sensors and Smart Structures Technologies for Civil, Mechanical, and Aerospace Systems, 2013, vol. 8692 , p. 869227

[12] T. Kamas, V. Giurgiutiu, and B. Lin, "Modeling and Experimentation of Thickness Mode E/M Impedance and Rayleigh Wave Propagation for Piezoelectric Wafer Active Sensors on Thick Plates," in Volume 1: Development and Characterization of Multifunctional Materials; Modeling, Simulation and Control of Adaptive Systems; Structural Health Monitoring; Keynote Presentation, 2014, p. V001T05A003.

[13] A. Kamal, V. Giurgiutiu, and B. Lin, "Predictive modeling of PWAS-coupled shear horizontal waves," in SPIE 2013 Smart Structure/NDE, Health Monitoring and Smart NDE of Structural and Biological Systems, 2013, p. 86950F.

[14] K. K. Kanazawa and J. G. Gordon, "The oscillation frequency of a quartz resonator in contact with a liquid," Anal. Chem. Acta, vol. 175, pp. 99-105, 1985.

[15] F. Josse and Z. Shana, "Analysis of shear horizontal surface waves at the boundary between a pieazoelectric crystal and a viscous fluid medium," $J$. Acoust. Soc. Am., pp. 978-984, 1988.

[16] E. Nwankwo and C. J. Durning, "Mechanical response of thickness-shear mode quartz-crystal resonators to linear viscoelastic fluids," Sensors Actuators A Phys., vol. 64, pp. 119-124, 1998.

[17] S. J. Martin, H. L. Bandey, R. W. Cernosek, A. R. Hillman, M. J. Brown, and L. Le, "EquivalentCircuit Model for the Thickness-Shear Mode Resonator with a Viscoelastic Film Near Film Resonance," vol. II, no. 1, pp. 141-149, 2000.

[18] Y. K. Suh and Y. H. Kim, "Effect of Overlayer Viscoelasticity on Resonant Characteristics of a Quartz Crystal Resonator," Jpn. J. Appl. Phys., vol. 49, no. 6, p. 065801, Jun. 2010.

[19] F. L. Guo and R. Sun, "Propagation of BleusteinGulyaev wave in $6 \mathrm{~mm}$ piezoelectric materials loaded with viscous liquid," Int. J. Solids Struct., vol. 45, no. 13, pp. 3699-3710, Jun. 2008.

[20] C. Schaschke, I. Fletcher, and N. Glen, "Density and Viscosity Measurement of Diesel Fuels at Combined High Pressure and Elevated Temperature," Processes, vol. 1, no. 2, pp. 30-48, 2013.

[21] T. Kamas, "Behavior of Piezoelectric Wafer Active Sensor in Various Media," University of South Carolina, 2014.

[22] “APC 850 material properties." [Online]. Available: www.americanpiezo.com. [Accessed: 29-Jun-2015].

[23] “ANSYS Help.” 2009.

[24] C. Schaschke, I. Fletcher, and N. Glen, "Density and Viscosity Measurement of Diesel Fuels at Combined High Pressure and Elevated Temperature," Processes, vol. 1, pp. 30-48, 2013. 